\title{
Grid Adaptation and Non-iterative Defect Correction for Improved Accuracy of Numerical Solutions of PDEs
}

\author{
M. Razi ${ }^{\mathrm{a}, *}$, P. J. Attar ${ }^{\mathrm{a}}$, P. Vedula ${ }^{\mathrm{a}}$ \\ ${ }^{a}$ School of Aerospace and Mechanical Engineering, The University of Oklahoma
}

\begin{abstract}
In this work we present a computational approach for improving the order of accuracy of a given finite difference method for solution of linear and nonlinear hyperbolic partial differential equations. The methodology consists of analysis of leading order terms in the discretization error of any given finite difference method, leading to a modified version of the original partial differential equation. Singular perturbations of this modified equation are regularized using an adaptive grid distribution and a non-iterative defect correction method is used to eliminate the leading order, regular perturbation terms in the modified equation. Implementation of this approach on a low order finite difference scheme not only results in an increase in its order of accuracy but also results in an improvement in its numerical stability due to the regularization of singular perturbations. The proposed approach is applied to four different canonical problems including the numerical solution of (1) Liouville equation, (2) inviscid Burgers equation, (3) nonlinear reaction-advection equation and a (4) system of hyperbolic PDEs. When compared to exact solutions, the numerical results demonstrate the ability of this method in both boosting the accuracy of finite difference schemes up to the desired order and also providing a fully stable numerical solution.
\end{abstract}

Keywords: adaptive finite difference solution; Burgers equation; hyperbolic equations; Liouville equation; non-iterative defect correction; numerical stability; reaction-advection equation; singularity adaptive grid.

*Corresponding Author. Mailing Address: 865 Asp Ave., Felgar Hall Rm 212A, Norman OK 73019-1052; Tel: +14055506943

Email addresses: mani.razi-1@ou.edu (M. Razi), peter.attar@ou.edu (P. J. Attar), pvedula@ou.edu (P. Vedula) 


\section{Introduction}

As analytical solutions of partial differential equations (PDEs) are available only in rare cases, numerical methods are widely used for solution of PDEs. Among these methods, high-order numerical schemes have been thoroughly investigated in recent times. Due to limitations of computational resources, especially needed for solutions of PDEs in multidimensional spaces, development of efficient high-order schemes has become an active area of research $[1,2,3,4,5]$.

Due to their conceptual simplicity, and ease of implementation, finite difference schemes $[6,7,8,9,10,11]$ are commonly used to obtain numerical solutions of linear and nonlinear partial differential equations. The objectives of many research efforts regarding the development of new finite difference methods include derivation of schemes which have high accuracy, suitable numerical stability and reasonable computational costs. As high order finite difference schemes typically are more complicated to develop and implement, when compared to lower order schemes, a number of methodologies have been developed which enhance the accuracy of underlying low-order schemes through choices made regarding the computational (spatial and temporal) grid. Such approaches are based upon reducing the truncation error of these schemes through application of various numerical techniques including adaptive grid refinement [12, 13], Richardson extrapolation [14], defect correction $[15,16,17]$ and optimal time-step selection combined with non-iterative defect correction (OTS-NIDC) [18].

As the truncation error of a finite difference scheme is a function of spatial and temporal increments [9], refining the spatial step size can directly enhance the accuracy of the numerical solution. Unfortunately refining the grid over the entire domain of computation is computationally expensive especially in a multidimensional space. On the other hand, adaptive refinement of the spatial grid is well suited for this purpose. Using this technique, a mesh is refined only in those regions of the computational domain where high resolution is most needed in terms of solution accuracy. This technique can be tailored based upon the type of partial differential equation to be solved.

As hyperbolic PDEs are often found in many disciplines including fluid dynamics [19, 20, 21, 22], acoustics [23, 24], electromagnetics [25], heat transfer [26] and some other areas of science and engineering, there is a need for efficient methods for improving the accuracy of numerical solutions. For nu- 
merical solution of PDEs with large variability in spatial gradients, it would appear desirable to employ a non-uniform mesh which adapts in time such that fine grid resolution is used in regions with large gradients and a more coarse resolution is used in smooth regions. The problem of mesh redistribution for hyperbolic partial differential equations has been addressed extensively in the literature starting with the work of Harten and Hyman[27] who employed a static regridding procedure, and more recently in the work of Stockie et al.[28], who used a semi-implicit approach which coupled moving mesh equations based upon the equidistribution principle to a high-resolution Godunov-type scheme for the physical PDE. As shown in [29], a grid point distribution is asymptotically optimal if some error measure is equally distributed over the field. This concept is the motivation for the use of the equidistribution, over the solution domain, of some monitor function (or its integral) to determine a mesh. In the moving mesh literature this is known as the equidistribution principle. Typical monitor functions which are used are the arclength and local curvature of the solution [30, 31].

While the adaptive mesh approach based upon equidistribution attempts to minimize the error in regions of strong gradients and local extrema, another possibility in reducing numerical error is to attempt to find a mesh distribution which equidistributes or minimizes the local truncation error or its estimate $[32,33,34,35,36]$. Using this principle Carey and Dinh [37] introduced an optimal grading function in various norms and semi-norms for two-point boundary-value problems. This approach improves the accuracy of a numerical solution for a given number of grid points through adaptive mesh redistribution in high gradient regions. Although, most of these methods do not require any prior truncation error analysis and provide the original low-order finite difference scheme with super convergent properties, non-uniformity of grids may introduce an additional source of error and some of these methods are difficult to extend to multiple dimensions. Moreover, as shown by Yamaleev [36], it is impossible to increase the global (formal) order of accuracy for first order finite difference schemes only by applying a grid distribution. The use of an adaptive grid distribution technique does not affect the temporal order of accuracy and also modifies the range of numerical stability for explicit schemes as it refines spatial increments, often resulting in the need to use very small time-steps.

Another popular approach for increasing the accuracy of lower-order schemes is through the use of Richardson extrapolation [38, 39]. This method improves the spatial order of accuracy by considering successive finite differ- 
ence computations on increasingly refined, equally spaced, meshes, followed by linear extrapolation on the computed results. However, this approach requires function evaluations at refined locations and prior truncation error analysis to obtain the linear extrapolation. Although this method can be applied to enhance both temporal and spatial orders of accuracy [14], the numerical stability on the finer grid is not guaranteed and, due to its significant computer memory usage, it is rarely extended to more than one spatial dimension.

Another effective way of reducing the truncation error of finite difference schemes is through the use of defect correction methods [40]. These methods are developed based upon either truncation error analysis or through the use of a higher order finite difference scheme in addition to the lower order base scheme. The former type of defect correction approaches can be classified into two major groups including high-order compact and iterative defect correction methods. In high-order compact approaches [41, 42], first the stationary part of the differential equation is considered and a compact high-order scheme is derived using the truncation error analysis. Next, the temporal derivative is added to the compact discretized equation as a source term. As such, this approach is implicit. Moreover, it only reduces the spatial discretization error and the temporal accuracy is only affected by the order of temporal discretization of the underlying low-order scheme [18].

The second type of defect/differed correction approaches are developed based upon the subtraction of the leading terms of the truncation error expression from the low-order discretized equation [40]. In this regard, Jones [43] evaluated these terms in the stationary condition and enhanced the numerical stability and accuracy of difference schemes by applying them in the original low-order discretized equation. Alternatively, using an iterative procedure in defect correction process provides a highly accurate numerical scheme with improved stability properties. An example of this iterative approach is given in the work of Christleib et al. [44], who applied a spectral differed correction approach by predicting the residual error and then correcting the numerical solution in an iterative process. Implementing their approach to solve ordinary differential equations (ODE), they could obtain numerical results with superior stability properties and high accuracy. The iterative differed correction strategy has typically been applied to an underlying low-order implicit finite difference solution to enhance either temporal or spatial order of accuracy $[15,17]$. When used to improve both, domain decomposition method must be used which makes the approach computation- 
ally more complicated and more difficult to extend to higher dimensions [16].

Another form of defect correction methods can be developed by first solving the PDE with a low-order finite difference scheme and then applying a higher order scheme in an iterative procedure to increase the numerical solution accuracy [45]. This approach is typically designed to be used with implicit schemes where in each iteration, a system of equations with matrix coefficients governed by the lower order scheme is solved. These matrix solutions are often very efficient which, when combined with the increased order of accuracy, typically leads to a more computational efficient scheme despite the need for iteration. However, the numerical stability of this approach is highly dependent upon the underlying higher order finite difference scheme and application of this approach to explicit schemes typically does not provide any computational benefits.

Recently, a non-iterative defect correction method in conjunction with the optimal time-step selection (OTS-NIDC) has been used by Chu [18] to increase the order of accuracy of finite difference schemes. In this method, the leading order terms in the truncation error expression are categorized into singular and regular perturbation terms. While the latter group of perturbation terms is eliminated by a simple subtraction from the original discretized equation, singular perturbation terms are eliminated through an optimal time-step selection. The optimal time-step is obtained by solving the equation derived from setting singular perturbations to zero. This equation must give a constant time-step size for the entire computational domain as it is not feasible to use a spatially variable time-step in the framework of standard finite difference computations. This constant time-step value can be obtained only for linear and semi-linear PDEs and thus for most nonlinear PDEs, order of accuracy can not be improved by using such an approach.

In the present work, in order to increase the order of accuracy of finite difference schemes, we propose a method based on analysis of leading order terms in the discretization error leading to the construction of a singularly perturbed modified differential equation. The proposed method involves (i) regularization of the singular perturbations of the modified equation via the use of grid adaptation and (ii) non-iterative defect correction for leading order regular perturbations of the modified equation.

The grid adaptation is designed such that the spatial grid and time-step are chosen based on a constrained minimization problem, where the objective function is a chosen global error measure that represents the influence of the leading order singular perturbation term in the truncation error, subject to 
constraints of numerical stability and geometric bounds. The proposed approach can be considered as an extension to OTS-NIDC for solving nonlinear hyperbolic PDEs. As singular perturbations are involved in imposing the numerical stability limits on the explicit finite difference solution, eliminating these singularities provides a numerical scheme with better stability properties. The proposed method is also applicable to finite difference solutions on both moving and fixed domains.

In this article, as a proof of concept, four canonical problems are solved. The first test problem involves the numerical solution of a first order hyperbolic equation with a spatially dependent drift. Next, in order to demonstrate the method for nonlinear hyperbolic PDEs, the methodology is applied to solve both the inviscid Burgers and a nonlinear reaction-advection equations. Finally, the proposed approach is also used to obtain numerical solution of a system of hyperbolic PDEs. When the proposed method is applied to the first order upwind scheme, the results indicate an improvement in the order of accuracy. In this context, the proposed method also appears to lack the severe numerical diffusion errors generally found in first order upwind scheme. In addition, this approach is found to produce numerical solutions which lack non-physical numerical oscillations. For the test problems investigated in this paper, this result will be most evident when the scheme is applied to a system of hyperbolic PDEs. It should also be noted that while only applied to one-dimensional problems in this paper, the approach can be extended to multiple dimensions, particularly when dimension splitting techniques such as explicit alternate directions (EAD) [46] and alternate directions implicit (ADI) [8] are used.

\section{Numerical Method}

\subsection{Theoretical foundation}

In this paper, we consider a first order hyperbolic PDE in the following general form:

$$
\frac{\partial f}{\partial t}+\frac{\partial(D(f, x, t) f)}{\partial x}=S(f, x, t)
$$

where $D$ and $S$ denote drift and reaction functions, respectively. Equation 1 is a nonlinear non-homogeneous PDE which can be solved through approximation of the temporal and spatial derivatives with $p$-th order accurate finite 
difference formulas: Eq. 2.

$$
\sum_{m=n-m_{1}}^{n+m_{2}} \beta_{m} f_{i}^{(m)}+\sum_{l=i-l_{1}}^{i+l_{2}} \alpha_{l} D_{l}^{(n)} f_{l}^{(n)}-S\left(f_{i}^{(n)}\right)=0
$$

where $\beta_{m}$ and $\alpha_{l}$ are coefficients that depend on the type of finite difference formula used to approximate the derivatives in Eq. 1. It is well known that the solution of finite difference equation given in Eq. 2, which approximately solves Eq. 1, is the exact solution of a different, infinite order partial differential equation often called the modified differential equation [47]. Using Taylor series, the modified equation corresponding to Eq. 2 can be derived and its subtraction from the original PDE gives the truncation error expression at grid point $x_{i}$ and time-step $n$ :

$$
\mathrm{TE}=C_{P}^{t}\left(\frac{\partial^{p+1} f}{\partial t^{p+1}}\right)_{i}^{n}(\Delta t)^{p}+C_{P}^{x}\left(\frac{\partial^{p+1}(D f)}{\partial x^{p+1}}\right)_{i}^{n}(\Delta x)^{p}+\mathcal{O}(\Delta t+\Delta x)^{p+1}
$$

where TE denotes the truncation error of the $p$-th order accurate finite difference approximation and $C_{P}^{t}$ and $C_{P}^{x}$ are functions of $\beta_{m}$ and $\alpha_{l}$, respectively. For a first order difference scheme, after elimination of all time-derivatives higher than order one in Eq. 3, the truncation error can be written as

$$
\begin{aligned}
\mathrm{TE}= & \left(C_{P}^{t} D_{i}^{n} \Delta t+C_{p}^{x} \Delta x\right)\left(\frac{\partial^{2}(D f)}{\partial x^{2}}\right)_{i}^{n}+C_{P}^{t} \Delta t \times \\
& \left\{\left(\frac{\partial S}{\partial t}\right)_{i}^{n}-\left(\frac{\partial}{\partial x}(D S)\right)_{i}^{n}+\left(\frac{\partial D}{\partial x} \frac{\partial(D f)}{\partial x}\right)_{i}^{n}-\right. \\
& \left.\left(\frac{\partial}{\partial x}\left(f \frac{\partial D}{\partial t}\right)\right)_{i}^{n}\right\}+\mathcal{O}(\Delta t+\Delta x)^{2}
\end{aligned}
$$

This truncation error analysis can be easily extended to higher order schemes.

The leading order terms in the truncation error expressions can be classified into singular and regular perturbations of the original partial differential equation [18]. A simple means of improving the order of accuracy of finite difference schemes, using information from the truncation error expression, is to subtract the complete leading order expression from the original discretized equation. If the derivative terms in the leading order error expression are 
then approximated with finite difference formulas of equal or higher order than those used to derive the original finite difference equation, the resulting scheme order will be increased by one. Unfortunately it has been found that inclusion of the singular perturbation terms, in the subtracted expression, typically results in a unstable numerical solution [15]. An iterative approach using an underlying implicit scheme has been suggested to resolve this issue $[15,17]$.

The singular perturbative terms in the truncation error expression for a $p$-th order finite difference scheme can be identified as having the form

$$
\Gamma(\Delta x, \Delta t)=C_{P}^{x}(\Delta x)^{p}+C_{P}^{t} G(D)(\Delta t)^{p},
$$

where $G$ is a function of drift $(D)$ in Eq. 1 . If $G$ is constant, the OTS-NIDC approach can be used. This first involves choosing a time-step which makes Eq. 5 identically zero thereby resulting in the elimination of the leading order singular perturbative expression. The remaining leading order terms in the truncation error are then subtracted from the original finite difference equation such that the regular perturbative expression is resolved. The final result of the OTS-NIDC approach is a stable numerical solution which has an order of accuracy which is increased by one when compared to the original finite difference equation. Unfortunately if $G(D)$ in Eq. 5 is spatially dependent, the OTS-NIDC approach can not be easily applied as the time-steps which result from the procedure are spatially dependent. An alternate way of dealing with this problem is to minimize the singular perturbation terms through proper selection of both temporal and local spatial increments. This is the approach which is presented in the current work.

\subsection{Finite Difference Scheme}

In this work the first order (in space and time) upwinding is chosen as the underlying finite difference whose order of accuracy will be improved through the combined grid adaptation and defect correction approach. Application of the upwinding scheme to Eq. 1 results in the finite difference equation:

$$
\begin{aligned}
& f_{i}^{n+1}=f_{i}^{n}+(\Delta t / \Delta x)\left(D_{i-1}^{n} f_{i-1}^{n}-D_{i}^{n} f_{i}^{n}\right)+S_{i}, \text { if } D_{i} \geq 0, \\
& f_{i}^{n+1}=f_{i}^{n}+(\Delta t / \Delta x)\left(D_{i}^{n} f_{i}^{n}-D_{i+1}^{n} f_{i+1}^{n}\right)+S_{i}, \text { if } D_{i}<0 .
\end{aligned}
$$

From Eq. $6 C_{P}^{t}$ and $C_{P}^{x}$ in Eq. 5 can be identified, for $D_{i} \geq 0$, as -0.5 and 0.5 , respectively and as $C_{P}^{t}=C_{P}^{x}=0.5$ for $D_{i}<0$. Also accordingly $G(D)$ in Eq. 5 can be identified as being equal to $D$. Based upon the heuristic stability 
analysis approach proposed by Warming and Hyett [47], if the coefficient of the lowest even-order derivative in the truncation error expression is positive, the finite difference scheme is numerically stable. Thus, this scheme is stable if the Courant-Friedrichs-Lewy number $\left(C F L=D_{i}^{n} \Delta t / \Delta x\right)$ is less than or equal to one.

\subsection{Adaptive grid generation}

Over the past several decades a number of different methodologies have been developed for grid adaptation in the context of solving partial differential equations $[12,48,49,50]$. Typically, these grid adaptation techniques are either based upon the equidistribution principle [37] or minimization of truncation error [33]. In this work the redistribution strategy which is proposed is aimed at eliminating the leading order singular perturbation terms, e.g. $\Gamma$ in Eq. 5, of the modified partial differential equation. The ideal case where the leading order terms are identically zero may not always be attainable, except in a selected classes of PDEs (e.g. one-dimensional wave equation, with a constant wave speed). Hence, minimization of a global error measure is sought, where the error measure is driven to a small quantity (e.g. less than a tolerance value at least smaller than a high-order error term following the leading order error term). In general this can be accomplished through the solution of a nonlinear, constrained optimization problem for the time-step $\Delta t^{n}$ and spatial increments $\Delta x_{i}^{n}$. Given the set of $M$ grid points at time $t^{n},\left\{x_{1}^{n}, x_{2}^{n}, \ldots, x_{M}^{n}\right\}$, along with the values of $f$ on this grid, $\mathbf{F}^{n}=\left\{f_{1}^{n}, f_{2}^{n}, \ldots, f_{M}^{n}\right\}$, this optimization problem can be defined as

$$
\left\{\begin{array}{c}
\min \|\boldsymbol{\Gamma}\|_{2}^{2} \\
\sum_{i=1}^{M-1} \Delta x_{i}^{n}=x_{M}^{n}-x_{1}^{n}, \\
x_{M}^{n}=x_{M}^{n-1}, \\
x_{1}^{n}=x_{1}^{n-1} \\
0<\Delta t^{n}<\delta_{t} \\
0<\Delta x_{i}^{n}<x_{M}^{n}-x_{1}^{n} \quad i=1,2,3, \ldots, M-1,
\end{array}\right.
$$

where

$$
\begin{aligned}
\Delta x_{i}^{n} & =x_{i+1}^{n}-x_{i}^{n} \\
\Gamma_{i} & \equiv \Gamma_{i}\left(\Delta x_{i}^{n}, D^{n}, \Delta t^{n}\right) \equiv \Delta x_{i}^{n}-\left|D_{i+1}^{n}\right| \Delta t^{n}, i=1,2,3, \ldots, M-1
\end{aligned}
$$

In Eq. 7, the symbol $\delta_{t}$ denotes the upper bound for $\Delta t^{n}$ which is set here to a value of 0.5 . Equation 7 is solved for the $M$ unknowns including $\Delta t^{n}$ 
and $x_{k}^{n}(k=1,2, \ldots, M-1)$ using a standard trust region algorithm designed to solve problems with linear bound constraints [51]. For the particular PDE and finite difference method (based on first order upwinding of Eq. 1) used in this work, the minimum of the objective function, $\left\|\Delta x_{i}^{n}-\left|D_{i+1}^{n}\right| \Delta t^{n}\right\|_{2}^{2}$, is always found to be less than a tolerance value of $10^{-13}$. Thus, the contribution of the singular perturbations to the leading order terms in the discretization error are neglected (or eliminated). In other words, the original singularly perturbed modified differential equation can be regularized (numerically; with reference to a small tolerance value) via the time adaptive spatial grid obtained from the constrained minimization approach described above.

If the domain of $x, \Omega$, is infinite, the problem is simplified to the determination of the grid points $x_{i}^{n}$ and time-step $\Delta t^{n}$ as

$$
\begin{aligned}
x_{i}^{n} & =x_{i+1}^{n}-\left|D_{i+1}^{n}\right| \Delta t^{n} \\
\Delta t^{n} & =\frac{1}{M} \sum_{i=1}^{M} \frac{\left|x_{M}^{n}-x_{1}^{n}\right|}{(M-1) D_{i}^{n}}
\end{aligned}
$$

The mesh generated with the general approach given in Eq. 9, or the simplified approach given in Eqs. 10 and 11, automatically satisfies the numerical stability condition (e.g. CFL $\leq 1$ ) and eliminates the leading order singular perturbative terms in the modified PDE. It should be noted, however, that a solution of Eq. 7 is only possible if $G(D)$ does not contain a fixed point in $\Omega$.

\subsection{Non-iterative defect correction}

In this work, similar to the OTS-NIDC approach [18], non-iterative defect correction is used to resolve regular perturbations in the modified differential equation. As the leading order singular perturbation terms are eliminated through the grid redistribution procedure outlined in section 2.3 , they need not be included in the defect correction which in turn significantly improves the numerical stability of the method. Singularities of the modified equation contribute significantly in the numerical stability of the proposed defect correction approach. Once the singular perturbations are (numerically) regularized through the grid redistribution, the remaining leading order terms in the truncation error expression are eliminated through subtraction from the original low-order discretized equation. 
As mentioned, the defect correction involves subtraction of the leading order regular perturbative terms from the original finite difference equation. These terms then need to be approximated using finite difference approximations. As the purpose of applying the defect correction technique is to improve the accuracy of the scheme, these finite difference approximations must be of the same, or higher order, accuracy as those used to derive the original finite difference equation. For instance, in the current work for enhanced accuracy of the first order upwind (see Eq. 6) scheme, first order spatial derivatives contained in the defect correction expression are approximated, on a nonuniform mesh, as:

$$
\left(\frac{\partial f}{\partial x}\right)_{i}=\frac{-\alpha^{2} f_{i-1}+f_{i}\left(\alpha^{2}-1\right)+f_{i+1}}{\Delta x_{i-1} \alpha(1+\alpha)}+\mathcal{O}\left(\Delta x_{i-1}^{2}\right)
$$

where $\alpha=\frac{\Delta x_{i}}{\Delta x_{i-1}}$. Specific forms of defect corrected finite difference equations will be given in the results section for the various test problems investigated.

The combination of the grid redistribution procedure and defect correction eliminates the entire leading order truncation error, resulting in a (at least) one order of magnitude improvement in both the spatial and temporal accuracy. While only one-dimensional problems are investigated in this work, extension of the methodology to multiple dimensions is possible and will be the subject of future investigation.

\subsection{Rezoning approach to numerical solutions on time-varying meshes}

In this work we will use a rezoning approach $[52,53]$ to approximate the solution of Eq. 1 using a finite difference solution on a time-varying mesh (see section 2.3).

Consider a sequence of times $t^{n}$ for $n=0,1, .$. , where the time-steps $\Delta t^{n}=t^{n+1}-t^{n}$ need not be equal. We define a time-varying partition (mesh) of $x$ as $\Omega(t)$ and allow for the possibility that the boundary of $\Omega(t)$, $S_{\Omega}(t)$, is also time-varying. In the rezoning approach, $\Omega(t)$ is considered to vary only at discrete time instants $t=t^{n}$ and as such the time-derivative in Eq. 1 need not be transformed.

In the rezoning method a new mesh $\tilde{\Omega}\left(t^{n}\right)$ is first generated for timestep $t^{n+1}$ (see section 2.3). Following the generation of this new mesh the PDE is integrated for the current step with the mesh held fixed. This time integration requires the approximate numerical solution for $f$ at time-step $t^{n}$, denoted as $\mathbf{F}^{n}=\left\{f_{1}^{n}, f_{2}^{n}, \ldots\right\}$, to be known on $\tilde{\Omega}\left(t^{n}\right)$. However since this 
solution is only known on $\Omega\left(t^{n}\right)$, an interpolated solution $\tilde{\mathbf{F}}^{n}=\left\{\tilde{f}_{1}^{n}, \tilde{f}_{2}^{n}, \ldots\right\}$ must be found on $\tilde{\Omega}\left(t^{n}\right)$. This interpolated solution is then used in the defect corrected finite difference equation used to advance the solution from step $n$ to step $n+1$. In this work a second order monotonicity preserving cubic Hermite interpolation is used to determine $\tilde{\mathbf{F}}^{n}[54]$.

\section{Results and Discussion}

\subsection{Test Problem 1: Liouville Equation}

The first test problem which is used to demonstrate the proposed methodology, involves the numerical solution of the Liouville equation $[55,56,57]$ :

$$
f_{t}+(D(x) f)_{x}=0 .
$$

In the Liouville equation the drift function, $D \equiv D(x)$, is only a function of the spatial component $x$, and there is no source term. After application of the first order upwind scheme to approximate the solution of Eq. 13, the leading order truncation error can be written as

$$
\mathrm{TE}=-\frac{1}{2}\left(D_{i}^{n} \Delta t \mp \Delta x_{i}\right)\left((D f)_{x x}\right)_{i}^{n}-\frac{\Delta t}{2}\left(D_{x}(D f)_{x}\right)_{i}^{n}+\mathcal{O}\left(\Delta t+\Delta x_{i}\right)^{2},
$$

for $D \geq 0$ and $D<0$, respectively, and $\Gamma_{i}=D_{i}^{n} \Delta t \mp \Delta x_{i}$. Next, applying the defect correction step on the original discretized equation gives:

$$
\begin{array}{r}
f_{i}^{n+1}=\tilde{f}_{i}^{n}+\left(\Delta t^{n} / \Delta x_{i-1}^{n}\right)\left(D_{i-1}^{n} \tilde{f}_{i-1}^{n}-D_{i}^{n} \tilde{f}_{i}^{n}\right)+\frac{\left(\Delta t^{n}\right)^{2}}{2}\left(\tilde{f}_{i}^{n}\left(\left(D_{x}\right)_{i}^{n}\right)^{2}+\right. \\
\left.D_{i}^{n} \frac{-\alpha^{2} \tilde{f}_{i-1}^{n}+\tilde{f}_{i}^{n}\left(\alpha^{2}-1\right)+\tilde{f}_{i+1}^{n}}{\Delta x_{i-1}^{n} \alpha(1+\alpha)}\left(D_{x}\right)_{i}^{n}\right), \quad \text { if } D_{i}^{n} \geq 0, \quad \text { (1) }
\end{array}
$$

and

$$
\begin{array}{r}
f_{i}^{n+1}=\tilde{f}_{i}^{n}+\left(\Delta t^{n} / \Delta x_{i}^{n}\right)\left(D_{i}^{n} \tilde{f}_{i}^{n}-D_{i+1}^{n} \tilde{f}_{i+1}^{n}\right)+\frac{\left(\Delta t^{n}\right)^{2}}{2}\left(\tilde{f}_{i}^{n}\left(\left(D_{x}\right)_{i}^{n}\right)^{2}+\right. \\
\left.D_{i}^{n} \frac{-\alpha^{2} \tilde{f}_{i-1}^{n}+\tilde{f}_{i}^{n}\left(\alpha^{2}-1\right)+\tilde{f}_{i+1}^{n}}{\Delta x_{i-1}^{n} \alpha(1+\alpha)}\left(D_{x}\right)_{i}^{n}\right), \quad \text { if } D_{i}^{n}<0,
\end{array}
$$


In this test problem framework, two different cases with linear $(D(x)=$ $-x)$ and nonlinear $\left(D(x)=x-x^{3}\right)$ drift functions are investigated. The initial condition is a Gaussian distribution defined as

$$
f(x, 0)=\frac{1}{\sqrt{2 \pi} \sigma} \exp \left(-\frac{(x-\mu)^{2}}{2 \sigma^{2}}\right),
$$

and the value of the function $f$ at the domain boundaries is zero. In Eq. 16, $\mu$ and $\sigma$ are the mean and standard deviation of the initial Gaussian distribution function, respectively. Here, $\mu=1$ and $\sigma=0.1$ for the case with the linear drift and $\mu=2$ and $\sigma=0.1$ for the second case. For both cases, the exact solution can be obtained through characteristic analysis with results given by

$$
f(x, t)=\frac{1}{\sigma \sqrt{2 \pi}} \exp \left(-\frac{(x \exp (t)-\mu)^{2}}{2 \sigma^{2}}\right) \exp (t),
$$

and

$$
\begin{aligned}
f(x, t)= & \frac{1}{\sigma \sqrt{2 \pi}} \exp \left(-\frac{1}{2 \sigma^{2}}\left(\frac{\exp (-t)}{\sqrt{\exp (-2 t)-1+x^{-2}}}-\mu\right)^{2}\right) \\
& \times\left|1-x^{2}(1-\exp (-2 t))\right|^{-3 / 2},
\end{aligned}
$$

for the linear and nonlinear drift functions, respectively. As the domain for the continuous problem is infinite, at each time-step the grid is found using Eqs. 10 and 11 and the time-dependent boundary $S_{\Omega}(t)$ is determined based upon the tails of the response [58, 55]. For comparison purposes, numerical solutions are also generated with the standard upwinding scheme on a uniform grid within $S_{\Omega}(t)$.

In order to determine the impact of application of this approach on the accuracy of the numerical solution, the L2 error of the response (with linear drift) for three different times, $t=1,3$ and 5 , is shown in Fig. 1 versus the number of grid points in $x$. In this work, for $M$ grid points in $x$, the normalized L2 error is obtained as:

$$
\epsilon(t)=\left\{\frac{\sum_{i=1}^{M}\left(f_{\text {exact }}\left(x_{i}, t\right)-f_{\text {numerical }}\left(x_{i}, t\right)\right)^{2}}{\sum_{i=1}^{M}\left(f_{\text {exact }}\left(x_{i}, t\right)\right)^{2}}\right\}^{1 / 2}
$$


For the case with nonlinear drift, and also for the other test problems presented in this paper, the numerical results are shown for only one time-step. Results shown in Figs 1 (for linear Drift) and 2 (for nonlinear drift) clearly indicate that the proposed method results in an improvement in the numerical solution up to a desired order of accuracy. In Fig. 3, the effect of successive modifications (first to second order and first to third order) on the first order upwind method using the proposed approach is shown at $t=1$ for the case with a linear drift. In order to obtain third order accuracy following expression is subtracted from Eq. 16:

$$
\begin{aligned}
\mathrm{TE}= & \frac{1}{6} \Delta t^{2}\left(f\left(D_{x}\right)^{3}\right)_{i}^{n}+\frac{2}{3} \Delta t^{2}\left(D D_{x} D_{x x}\right)_{i}^{n}+\frac{7}{6} \Delta t^{2}\left(D D_{x}^{2} f_{x}\right)_{i}^{n}+ \\
& \frac{1}{6} \Delta t^{2}\left(D^{2} D_{x x} f_{x}\right)_{i}^{n}+\frac{1}{2} \Delta t^{2}\left(D^{2} D_{x} f_{x x}\right)_{i}^{n} .
\end{aligned}
$$

In addition to demonstrating that successive orders of improvement are possible with the current method, this figure shows that unlike the standard upwind solution, which approaches its formal first order accuracy gradually as the number of grid points increase, the solution obtained from the proposed method exhibits formal second or third order accuracy even with very few grid points. This result further indicates that the modified difference scheme is significantly less diffusive. From Fig. 3 it appears that the magnitude of the error improves by a (slighter) larger factor when going from first to second order when compared to the result of going from second to third order.

While standard linear upwinding finite difference schemes of order higher than one are known to be numerically unstable for solution of the advection equation $[46,8]$, the outcome of the approach presented in this paper is a stable numerical solution with a desired order of accuracy. While second order methods which employ nonlinear finite difference schemes, such as essentially non-oscillatory (ENO) [59] and TVD MacCormack [46] schemes, also result in stable numerical approximations, the current approach is simpler to implement and does not require the use of flux or slope limiters to avoid spurious oscillations in the response. It should also be noted that, as shown by Yamaleev [36], gradient (equidistribution) based refinement schemes can not be used to improve the order of accuracy for the first order finite difference schemes. 


\subsection{Test Problem 2: Inviscid Burgers Equation}

Burgers equation is a well-known model equation in fluid dynamics [8]. In its inviscid form, this equation is a nonlinear homogeneous hyperbolic PDE and can be written as:

$$
f_{t}+\left(\frac{f^{2}}{2}\right)_{x}=0
$$

Applying the first order upwind method, the original low-order discretized equation is given by:

$$
\left\{\begin{array}{cc}
f_{i}^{n+1}=f_{i}^{n}+\left(\frac{\Delta t^{n}}{2 \Delta x_{i-1}^{n}}\right)\left(\left(f_{i-1}^{n}\right)^{2}-\left(f_{i}^{n}\right)^{2}\right), & \text { if } f_{i}^{n} \geq 0 \\
f_{i}^{n+1}=f_{i}^{n}+\left(\frac{\Delta t^{n}}{2 \Delta x_{i}^{n}}\right)\left(\left(f_{i}^{n}\right)^{2}-\left(f_{i+1}^{n}\right)^{2}\right), & \text { if } f_{i}^{n}<0
\end{array}\right.
$$

where we recognize that $D$ and $S$ from Eq. 6 are $\frac{f^{2}}{2}$ and $S=0$ respectively. Thus, the truncation error expression can be derived from Eq. 5 as:

$$
\begin{aligned}
\mathrm{TE}= & \frac{f_{i}^{n}}{2}\left(f_{x x}\right)_{i}^{n}\left(\Delta x_{i-1}^{n}-f_{i}^{n} \Delta t^{n}\right)+\left(\frac{\Delta x_{i-1}^{n}}{2}-f_{i}^{n} \Delta t^{n}\right)\left(\left(f_{x}\right)_{i}^{n}\right)^{2}+ \\
& \mathcal{O}\left(\Delta t^{n}+\Delta x_{i-1}^{n}\right)^{2}
\end{aligned}
$$

and

$$
\begin{aligned}
\mathrm{TE}= & -\frac{f_{i}^{n}}{2}\left(f_{x x}\right)_{i}^{n}\left(\Delta x_{i}^{n}+f_{i}^{n} \Delta t^{n}\right)-\left(\frac{\Delta x_{i}^{n}}{2}+f_{i}^{n} \Delta t^{n}\right)\left(\left(f_{x}\right)_{i}^{n}\right)^{2}+ \\
& \mathcal{O}\left(\Delta t^{n}+\Delta x_{i}^{n}\right)^{2},
\end{aligned}
$$

for $f_{i}^{n} \geq 0$ and $f_{i}^{n}<0$, respectively. The terms used to redistribute the grid are $\Gamma_{i}=\left(\Delta x_{i-1}-f_{i}^{n} \Delta t^{n}\right)$ and $\Gamma_{i}=\left(\Delta x_{i}+f_{i}^{n} \Delta t^{n}\right.$ for $f_{i}^{n} \geq 0$ and $f_{i}^{n}<0$, respectively. Subtraction of the remaining (regular perturbative) leading order truncation terms from Eq. 23 gives

$$
\begin{aligned}
f_{i}^{n+1}= & \tilde{f}_{i}^{n}+\left(\frac{\Delta t^{n}}{2 \Delta x_{i-1}^{n}}\right)\left(\left(\tilde{f}_{i-1}^{n}\right)^{2}-\left(\tilde{f}_{i}^{n}\right)^{2}\right)-\Delta t^{n}\left(\frac{\Delta x_{i-1}^{n}}{2}-\tilde{f}_{i}^{n} \Delta t^{n}\right) \times \\
& \left(\frac{-\alpha^{2} \tilde{f}_{i-1}^{n}+\tilde{f}_{i}^{n}\left(\alpha^{2}-1\right)+\tilde{f}_{i+1}^{n}}{\Delta x_{i-1}^{n} \alpha(1+\alpha)}\right)^{2}, \text { if } \tilde{f}_{i}^{n} \geq 0
\end{aligned}
$$




$$
\begin{aligned}
f_{i}^{n+1}= & \tilde{f}_{i}^{n}+\left(\frac{\Delta t^{n}}{2 \Delta x^{n}}\right)\left(\left(\tilde{f}_{i}^{n}\right)^{2}-\left(\tilde{f}_{i+1}^{n}\right)^{2}\right)+\Delta t^{n}\left(\frac{\Delta x^{n}}{2}+\tilde{f}_{i}^{n} \Delta t^{n}\right) \times \\
& \left(\frac{-\alpha^{2} \tilde{f}_{i-1}^{n}+\tilde{f}_{i}^{n}\left(\alpha^{2}-1\right)+\tilde{f}_{i+1}^{n}}{\Delta x_{i-1}^{n} \alpha(1+\alpha)}\right)^{2}, \text { if } \tilde{f}_{i}^{n}<0
\end{aligned}
$$

For this test problem, the initial velocity profile at $t=0$ is defined to be:

$$
\left\{\begin{array}{c}
f(x, 0)=3-\cos (x), \text { if } 0 \leq x \leq 2 \pi, \\
f(x, 0)=2, \text { if } 2 \pi<x \leq 8
\end{array}\right.
$$

in the domain of computation which is bounded within $x=0$ and $x=8$ and the boundary conditions for $f$ are given as

$$
f(0, t)=f(8, t)=2 .
$$

The exact solution to Eqs. 22, with boundary conditions given by Eq. 29, is

$$
f(x, t)=3-\cos (x-f(x, t) t) .
$$

As the domain of computation is bounded for this problem, the grid at each time-step is generated based upon the nonlinear constrained optimization problem defined in Eq. 7. Using the set of grid point locations from the previous time-step as the initial guess for the trust-region algorithm, the number of iterations necessary to reach convergence was found to be less than 10. In this algorithm, the iterations are converged if any of the following values are smaller than predefined tolerances $\left(\varepsilon_{1}, \varepsilon_{2}, \ldots, \varepsilon_{6}\right)$ : the trust region area $\Delta$, L2 norm of the functional values $\|f\|_{2}$, L2 norm of the Jacobian matrix $\|J\|_{2}$, the trial step size of the iterations $\lambda$ or the expression $\|f\|_{2}-\|f-J \times S\|_{2}$. In this work, the tolerance $\varepsilon_{1}$ is set to $10^{-10}$ and the remaining convergence criteria (or tolerances) are fixed at a value smaller than machine precision.

Due to the nonlinear and hyperbolic nature of the Burgers equation, the solution evolves to a shock discontinuity. The development, over time, of this discontinuity is shown in Fig. 4. Setting the solution of the (nonlinear) Eq. 30, obtained with the Newton-Raphson method, as the reference solution, the L2 error is computed. The L2 error for the original and modified schemes (second order and third order), at $t=0.8$, are shown in Fig. 5. In order to obtain the third order accuracy, the following truncation error terms need to be removed using the non-iterative defect correction method:

$$
\mathrm{TE}=\Delta t^{2}\left(f \times f_{x}^{3}\right)_{i}^{n}+\Delta t^{2}\left(f^{2} f_{x} f_{x x}\right)_{i}^{n} .
$$


Along with demonstrating that the desired order of accuracy can be achieved, the results shown in Fig 5 also indicate that the third order scheme has almost an order of magnitude improvement in the magnitude of the error when compared to the third order ENO scheme.

\subsection{Test Problem 3: Nonlinear Reaction-Advection Equation}

The third test problem in this paper consists of the numerical solution of a nonlinear non-homogeneous hyperbolic PDE which represents logistic population growth coupled with an advective process. The differential equation which governs the time evolution of the scaled, strictly positive, population density $\rho$, which contains both reaction and drift functions, is given by [60]:

$$
\rho_{t}-\left(\rho^{2} / 2\right)_{x}=\rho(1-\rho)
$$

with an initial distribution for $\rho$ given as:

$$
\rho(x, 0)=\frac{1}{2}\left[2+\mathrm{e}^{x}+\mathrm{e}^{\frac{x}{2}} \sqrt{4+\mathrm{e}^{x}}\right] .
$$

Eq. 32 is solved on a fixed domain of computation $x \in[0,1]$, where the time-dependent boundary conditions are given as:

$$
\begin{gathered}
\rho(0, t)=\frac{\mathrm{e}^{-t}}{2}\left[2 \mathrm{e}^{t}+1+\sqrt{4 \mathrm{e}^{t}+1}\right] . \\
\rho(1, t)=\frac{\mathrm{e}^{-t}}{2}\left[2 \mathrm{e}^{t}+\mathrm{e}+\mathrm{e}^{\frac{1}{2}} \sqrt{4 \mathrm{e}^{t}+\mathrm{e}}\right] .
\end{gathered}
$$

After considerable algebra, the exact solution of Eq. 32, with the corresponding initial and boundary conditions, is obtained as

$$
\rho(x, t)=\frac{\mathrm{e}^{-t}}{2}\left[2 \mathrm{e}^{t}+\mathrm{e}^{x}+\mathrm{e}^{\frac{x}{2}} \sqrt{4 \mathrm{e}^{t}+\mathrm{e}^{x}}\right] .
$$

As in the previous problems, the first order upwind scheme is applied to approximate the solution of Eq. 32 and the expression for the truncation error is given by:

$$
\begin{aligned}
\mathrm{TE}= & \left(\frac{1}{2}\right)\left(\Delta x_{i}^{n}-\rho_{i}^{n} \Delta t^{n}\right) \rho_{i}^{n}\left(\rho_{x x}\right)_{i}^{n}+\left(\frac{\Delta x_{i}^{n}}{2}-\rho_{i}^{n} \Delta t^{n}\right)\left(\left(\rho_{x}\right)_{i}^{n}\right)^{2}+ \\
& \frac{\Delta t^{n}}{2}\left\{\rho_{i}^{n}\left(\rho_{x}\right)_{i}^{n}\left(3-5 \rho_{i}^{n}\right)-3\left(\rho_{i}^{n}\right)^{2}+2\left(\rho_{i}^{n}\right)^{3}+\rho_{i}^{n}\right\}+ \\
& \mathcal{O}\left(\Delta t^{n}+\Delta x_{i}^{n}\right)^{2} .
\end{aligned}
$$


From Eq. 37 we identify the term used to redistribute the grid as:

$$
\Gamma_{i}=\Delta x_{i}^{n}-\rho_{i}^{n} \Delta t^{n}
$$

Based upon the modified equation approach proposed by Warming and Hyett [47], which gives the necessary condition for numerical stability, it is found that the numerical solution is stable only if $\Gamma_{i}$ is positive, e.g. the local CFL number must be less than one. After subtraction of the leading order regular perturbative expression from the original low-order finite difference equation we arrive at the finite difference equation used to solve for $\rho_{i}$ and time-step $n+1$ :

$$
\begin{aligned}
\rho_{i}^{n+1}= & \tilde{\rho}_{i}^{n}+\left(\frac{\Delta t^{n}}{2 \Delta x_{i}^{n}}\right)\left(\left(\tilde{\rho}_{i}^{n}\right)^{2}-\left(\tilde{\rho}_{i+1}^{n}\right)^{2}\right)+\Delta t^{n} \tilde{\rho}_{i}^{n}\left(1-\tilde{\rho}_{i}^{n}\right)- \\
& \Delta t^{n}\left(\frac{-\alpha^{2} \tilde{\rho}_{i-1}^{n}+\tilde{\rho}_{i}^{n}\left(\alpha^{2}-1\right)+\tilde{\rho}_{i+1}^{n}}{\Delta x_{i-1}^{n} \alpha(1+\alpha)}\right)^{2}\left(\frac{\Delta x_{i}^{n}}{2}-\tilde{\rho}_{i}^{n} \Delta t^{n}\right)+ \\
& \left(\frac{\left(\Delta t^{n}\right)^{2}}{2}\right)\left(\tilde{\rho}_{i}^{n} \frac{-\alpha^{2} \tilde{\rho}_{i-1}^{n}+\tilde{\rho}_{i}^{n}\left(\alpha^{2}-1\right)+\tilde{\rho}_{i+1}^{n}}{\Delta x_{i-1}^{n} \alpha(1+\alpha)} \times\right. \\
& \left.\left(3-5 \tilde{\rho}_{i}^{n}\right)+\tilde{\rho}_{i+1}^{n}+2\left(\tilde{\rho}_{i+1}^{n}\right)^{3}-3\left(\tilde{\rho}_{i+1}^{n}\right)^{2}\right), \quad \tilde{\rho}_{i}^{n} \geq 0 .
\end{aligned}
$$

In Eq. 39, we have approximated the first derivative terms contained in the regular perturbative expressions using Eq. 12. As demonstrated in the previous problems, additional enhancement (beyond second order) can be obtained by removing higher order truncated error terms in a non-iterative defect correction process. For instance, in order to obtain the third order accuracy for the current problem the following terms should be subtracted from Eq. 39 after a proper discretization:

$$
\begin{aligned}
\mathrm{TE}= & -\Delta t^{2}\left(\frac{1}{6} \rho-\frac{7}{6} \rho^{2}+2 \rho^{3}-\rho^{4}+\frac{7}{6} \rho \rho_{x}-5 \rho^{3} \rho_{x}+\right. \\
& \frac{13}{3} \rho^{3} \rho_{x}+2 \rho \rho_{x}^{2}-\frac{9}{2} \rho^{2} \rho_{x}^{2}+\rho \rho_{x}^{3}+ \\
& \left.\rho^{2} \rho_{x x}-\frac{3}{2} \rho^{3} \rho_{x x}+\rho^{2} \rho_{x}^{2} \rho_{x x}\right)
\end{aligned}
$$


If the finite difference computations are performed on an adaptive grid defined through Eq. 7, with $\Gamma_{i}$ defined in Eq. 38, the finite difference scheme results in a stable numerical solution with the desired order of accuracy. This result is demonstrated in Figs. 6 and 7 where it can be seen that the numerical results obtained with the proposed method are second and third order accurate in both space and time (in contrast to the base first order upwind method). As the temporal increment is variable in time, an averaged value $\left(\Delta t_{\text {average }}\right)$ is considered in Fig 7 when verifying the temporal order of accuracy. Also, similar to the previous test problems, in order to compute the L2 error the exact solution is considered as the reference value.

\subsection{Test Problem 4: A System of Hyperbolic PDEs}

The last canonical problem in this paper is devoted to the numerical solution of a system of one-dimensional hyperbolic PDEs using the proposed methodology. The system of PDEs considered in this test problem consists of two hyperbolic PDEs and it arises in the study of small transverse vibration of string and rod with variable density and Young's modulus, respectively [61]. The dynamics of such a problem is governed by the second order wave equation (in non-dimensional form) with space-dependent wave propagation speed:

$$
U_{t t}-c^{2}(x) U_{x x}=0
$$

Equation 41 can be written as a system of two linear advection equations with a variable propagation speed:

$$
\left\{\begin{array}{c}
V_{t}=U_{x} \\
U_{t}=c^{2}(x) V_{x}
\end{array}\right.
$$

In this one-dimensional example problem, $c(x)=x+1$ and the initial conditions for both variables are given as:

$$
\left\{\begin{array}{c}
U(x, 0)=\frac{1}{\sqrt{(2 \pi)}} \exp \left(\frac{(x-20)^{2}}{2}\right), \\
V(x, 0)=0,0 \leq x \leq 60
\end{array}\right.
$$

Also, the boundary conditions for this bounded domain are defined as

$$
U(0, t)=U(60, t)=V(0, t)=V(60, t)=0 .
$$


For this problem the second order MacCormack scheme [9] is used as the underlying scheme which is modified. As the exact solution is not available, a highly refined $\left(M=6.4 \times 10^{4}\right.$ grid points) numerical solution (standard MacCormack) is used as the basis for comparison. Application of the MacCormack scheme to Eq. 42 gives:

$$
\begin{aligned}
V_{i}^{n+1}= & V_{i}^{n}+(\Delta t / 2 \Delta x)\left(U_{i+1}^{n}-U_{i-1}^{n}\right)+\left(\Delta t^{2} c_{i}^{2} / 2 \Delta x^{2}\right) \times \\
& \left(V_{i+1}^{n}-2 V_{i}^{n}+V_{i-1}^{n}\right), \\
U_{i}^{n+1}= & U_{i}^{n}+\left(\Delta t c_{i}^{2} / 2 \Delta x\right)\left(V_{i+1}^{n}-V_{i-1}^{n}\right)+\left(\Delta t^{2} c_{i}^{2} / 2 \Delta x^{2}\right) \times \\
& \left(U_{i+1}^{n}-2 U_{i}^{n}+U_{i-1}^{n}\right) .
\end{aligned}
$$

The truncation error expressions for these equations are obtained by the method of modified equations as

$$
\begin{aligned}
\mathrm{TE}^{(V)}= & -\Delta t c_{x} c V_{x}+\frac{\Delta t^{2}}{6} c_{x} c U_{x x}+\frac{1}{6}\left(\Delta x^{2}-\Delta t^{2} c^{2}\right) U_{x x x}+ \\
& \mathcal{O}\left(\Delta t^{n}+\Delta x^{n}\right)^{3} \\
\mathrm{TE}^{(U)}= & \frac{\Delta t^{2}}{6}\left(c^{2} c_{x}^{2} V_{x}+c^{3} c_{x x} V_{x}-c^{3} c_{x} V_{x x}\right)+\frac{c^{2}}{6}\left(\Delta x^{2}-\Delta t^{2} c^{2}\right) V_{x x x}+ \\
& \mathcal{O}\left(\Delta t^{n}+\Delta x^{n}\right)^{3} .
\end{aligned}
$$

Here, the proposed methodology is used to first eliminate the implicit numerical dispersion (coefficients of $U_{x x x}$ and $V_{x x x}$ in Eq. 46) through grid adaptation. The remaining leading order terms in the truncation error expression are then removed in a non-iterative defect correction process. In order to account for the non-uniformity of the grids, the objective function, $\Gamma$, is found to be

$$
\Gamma_{i}=\left(\Delta x_{i}-c_{i} \Delta t\right)\left(\frac{-1+3 \alpha}{1+\alpha}\right)^{\frac{1}{2}} .
$$

As shown in Figs. 8 and 9, which gives results for the modified third order scheme with $M=100$ and $M=200$, the numerical solution obtained from the proposed approach agrees well with the corresponding highly resolved numerical solution. The results also indicate that the numerical characteristics (accuracy and non-physical oscillation) of the current method are much improved when compared to the standard MacCormack scheme. 


\section{Conclusion}

In this work a novel approach is introduced to improve the formal order of accuracy of finite difference solutions to linear and nonlinear hyperbolic partial differential equations (PDEs). The proposed approach, which consists of two separate steps, is based upon analysis of the truncation error of the underlying finite difference equation. The first step involves the determination of a computational grid through a nonlinear, constrained optimization problem. When a solution to this optimization problem exists, the result is the elimination of the leading order singular perturbation terms in the modified differential equation. This grid distribution technique eliminates the need for an iterative defect correction process and is applicable to both moving (when the underlying continuous domain is infinite) and fixed domains of computation. When the hyperbolic PDE is solved on a moving domain, the grid distribution problem is reduced to the solution of a system of linear equations. The second step in the method is the removal of the remaining leading order (regular) perturbative terms in the modified differential equation through non-iterative defect correction approach. Here, the original low-order discretized equation is modified to include the leading terms in the truncation error expression corresponding to regular perturbations. The result of these two steps is a scheme which has (at least) one order higher accuracy than that given by the original finite difference equation.

In order to demonstrate the method, four one-dimensional benchmark test problems were solved. In this paper, both the first order upwind scheme and the second order MacCormack scheme were used as the underlying low-order scheme to be modified in the manner described in the previous paragraph. The first problem examined was the numerical solution of the Liouville equation which is a linear PDE. For this problem, cases with linear and nonlinear spatially dependent drift functions were studied. The second and third test problems were concerned with enhancing the accuracy of finite difference solutions of homogeneous and non-homogeneous nonlinear advection equations. For each of these example problems comparison with the exact solution indicates that the desired order of accuracy enhancement in the results is obtained when the proposed approach is used. The last canonical problem investigated dealt with the numerical solution of a system of linear hyperbolic PDEs with spatially dependent wave speed. The proposed scheme was shown to agree well with a highly refined solution and was much improved over the underlying (second order) scheme. 
Overall the results from each of the test problems indicate that the proposed method is able achieve a stable numerical solution, free from nonphysical numerical oscillation, with a desired (designed) formal order of accuracy. Unlike many standard schemes, due to small numerical diffusion, this formal order of accuracy can be achieved with very few grid points. While only one-dimensional problems were studied in this article, the proposed methodology can be extended to multidimensional problems which use either standard or dimension splitting finite difference schemes. This will be investigated in future work along with application of the method for problems in both fluid and structural dynamics.

\section{Acknowledgments}

The authors would like to acknowledge the financial support provided by NSF Award Numbers CMMI-1301093 and CBET-133700 and the University of Oklahoma Supercomputing Center (OSCER) which provided supercomputing time to the authors enabling them in the completion of this work.

\section{References}

[1] F. Bade, P. Haldenwang, High order scheme for thermally driven flows in an open channel, Computers \& fluids 27 (1998) 273-290.

[2] T. P. Loc, R. Bouard, Numerical solution of the early stage of the unsteady viscous flow around a circular cylinder: A comparison with experimental visualization and measurements, Journal of Fluid Mechanics 160 (1985) 93-117.

[3] X. Zhong, M. Tatineni, High-order non-uniform grid schemes for numerical simulation of hypersonic boundary-layer stability and transition, Journal of Computational Physics 190 (2003) 419-458.

[4] W. Cai, High-order hybrid numerical simulations of two-dimensional detonation waves, AIAA journal 33 (1995) 1248-1255.

[5] A. Rezgui, P. Cinnella, A. Lerat, Third-order accurate finite volume schemes for Euler computations on curvilinear meshes, Computers \& fluids 30 (2001) 875-901. 
[6] F. G. Blottner, Finite difference methods of solution of the boundarylayer equations, AIAA Journal 8 (1970) 193-205.

[7] S. W. Armfield, Finite difference solutions of the Navier-Stokes equations on staggered and non-staggered grids, Computers \& Fluids 20 (1991) 1-17.

[8] J. C. Tannehill, D. D. A. Anderson, H. Pletcher, R, Computational fluid mechanics and heat transfer, Taylor \& Francis, 1997.

[9] J. D. Hoffman, Numerical methods for engineers and scientists, McGrawHill, New York, USA, 1992.

[10] A. Bermudez, M. E. Vazquez, Upwind methods for hyperbolic conservation laws with source terms, Computers \& Fluids 23 (1994) 1049-1071.

[11] J. L. Steger, Implicit finite-difference simulation of flow about arbitrary two-dimensional geometries, AIAA Journal 16 (1978) 679-686.

[12] P. R. Eiseman, Adaptive grid generation, Computer Methods in Applied Mechanics and Engineering 64 (1987) 321-376.

[13] M. J. Berger, J. Oliger, Adaptive mesh refinement for hyperbolic partial differential equations, Journal of computational Physics 53 (1984) 484512 .

[14] S. A. Richards, Completed Richardson extrapolation in space and time, Communications in numerical methods in engineering 13 (1997) 573582.

[15] W. Kress, Error estimates for deferred correction methods in time, Applied numerical mathematics 57 (2007) 335-353.

[16] B. Gustafsson, L. Hemmingsson-Frändén, Deferred correction in space and time, Journal of scientific computing 17 (2002) 541-550.

[17] B. Gustafsson, L. Hemmingsson-Frändén, Implicit high-order difference methods and domain decomposition for hyperbolic problems, Applied Numerical Mathematics 33 (2000) 493-500. 
[18] K. T. Chu, Boosting the accuracy of finite difference schemes via optimal time step selection and non-iterative defect correction, Applied Mathematics and Computation 218 (2011) 3596-3614.

[19] S. Benzoni-Gavage, D. Serre, Multi-dimensional hyperbolic partial differential equations, Clarendon Press Oxford, 2007.

[20] T. Nishida, Nonlinear hyperbolic equations and related topics in fluid dynamics, Université de Paris-Sud, Département de Mathématique, 1978.

[21] U. Harlander, L. R. Maas, Two alternatives for solving hyperbolic boundary value problems of geophysical fluid dynamics, Journal of Fluid Mechanics 588 (2007) 331.

[22] Y. Ren, Q. Liu, S. Wang, M. Shen, A high order accurate, non-oscillating finite volume scheme using spline interpolation for solving hyperbolic conservation laws (computational fluid dynamics), Acta Aerodynamica Sinica 14 (1996) 281-287.

[23] G. Avalos, The exponential stability of a coupled hyperbolic/parabolic system arising in structural acoustics, in: Abstract and Applied Analysis, volume 1, Hindawi Publishing Corporation, 1996, pp. 203-217.

[24] J. Hunter, Hyperbolic waves and nonlinear geometrical acoustics, in: Trans. 6th Army Conference on Appl. Math. and Computing, volume 2, 1989, pp. 527-569.

[25] A. Majda, M. Taylor, Inverse scattering problems for transparent obstacles, electromagnetic waves, and hyperbolic systems, Communications in Partial Differential Equations 2 (1977) 395-438.

[26] W. Kaminski, Hyperbolic heat conduction equation for materials with a nonhomogeneous inner structure, Journal of Heat Transfer (Transactions of the ASME (American Society of Mechanical Engineers), Series C);(United States) 112 (1990).

[27] A. Harten, J. M. Hyman, Self-adjusting grid methods for onedimensional hyperbolic conservation laws, J. Comput. Phys 50 (1981) 235-269. 
[28] J. M. Stockie, J. A. Mackenzie, R. D. Russell, A moving mesh method for one-dimensional hyperbolic conservation laws, SIAM J. Sci. Comput. 22 (2001) 1791-1813.

[29] I. Babuška, W. C. Rheinboldt, A-posteriori error estimates for the finite element method, Int. J. Numer. Methods Engrg. 12 (1978) 1597-1615.

[30] A. B. White, On selection of equidistributing meshes for two-point boundary problems, SIAM J. Numer. Anal. 16 (1979) 472-502.

[31] H. A. Dwyer, Grid adaptation for problems in fluid dynamics, AIAA J. 22 (1984) 1705-1712.

[32] M. Letini, V. Pereyra, An adaptive finite difference solver for nonlinear two-point boundary problems with mild boundary layers, SIAM J. Numer. Anal. 4 (1977) 91-111.

[33] G. H. Klopfer, D. S. McRae, The nonlinear modified equation approach to analyzing finite difference scheme, Paper No. 81-1029, AIAA, 1981.

[34] V. E. Denny, R. B. Landis, A new method for solving two-point boundary-value problems using optimal node distribution, J. Comput. Phys. 9 (1972) 120-137.

[35] B. Pierson, P. Kutler, Optimal nodal point distribution for improved accuracy in computational fluid dynamics, AIAA J. 18 (1980) 49-54.

[36] N. K. Yamaleev, Minimization of the truncation error by grid adaptation, Report No. 99-461999, ICASE, 1999.

[37] G. F. Carey, H. T. Dinh, Grading functions and mesh redistribution, SIAM Journal on Numerical Analysis 22 (1985) 1028-1040.

[38] A. Sidi, Practical extrapolation methods: Theory and applications, Cambridge University Press, 2003.

[39] W. Shyy, M. Garbey, A. Appukuttan, J. Wu, Evaluation of Richardson extrapolation in computational fluid dynamics, Numerical Heat Transfer: Part B: Fundamentals 41 (2002) 139-164. 
[40] R. D. Skeel, A theoretical framework for proving accuracy results for deferred corrections, SIAM Journal on Numerical Analysis 19 (1982) $171-196$.

[41] W. F. Spotz, G. F. Carey, High-order compact finite difference methods, in: Preliminary Proceedings International Conference on Spectral and High Order Methods, Houston, TX, 1995.

[42] W. F. Spotz, G. F. Carey, Extension of high-order compact schemes to time-dependent problems, Numerical Methods for Partial Differential Equations 17 (2001) 657-672.

[43] D. A. Jones, Modified-truncation finite difference schemes, Journal of Computational Physics 209 (2005) 322-339.

[44] A. Christlieb, B. Ong, J. Qiu, Integral deferred correction methods constructed with high order Runge-Kutta integrators, Mathematics of Computation 79 (2010) 761-783.

[45] P. Wesseling, Principles of Computational Fluid Dynamics, Springer, 2009.

[46] V. Guinot, Wave propagation in fluids: Models and numerical techniques, John Wiley \& Sons, Hoboken, New Jersey, USA, 2010.

[47] R. F. Warming, B. J. Hyett, The modified equation approach to the stability and accuracy analysis of finite-difference methods, Journal of Computational Physics 14 (1974) 159-179.

[48] J. F. Thompson, B. K. Soni, N. P. Weatherill, Handbook of Grid Generation, Taylor \& Francis, 1998.

[49] V. D. Liseikin, Grid Generation Methods, Scientific computation, Springer, 2009.

[50] G. F. Carey, Computational Grids: Generations, Adaptation \& Solution Strategies, Series in Computational and Physical Processes in Mechanics, Taylor \& Francis, 1997.

[51] A. R. Conn, N. I. M. Gould, P. L. Toint, Trust Region Methods, Society for Industrial and Applied Mathematics, 2000. 
[52] W. Huang, R. D. Russell, Adaptive moving mesh methods, Springer, New York, USA, 2011.

[53] C. J. Budd, W. Huang, R. D. Russell, Adaptivity with moving grids, Acta Numerica 18 (2009) 111-241.

[54] J. M. Hyman, Accurate monotonicity preserving cubic interpolation, SIAM J. Sci. Comput 4 (1983) 645-654.

[55] M. Razi, P. J. Attar, P. Vedula, Uncertainty quantification of multidimensional dynamical systems based on adaptive numerical solutions of the Liouville equation, Submitted to Probabilistic Engineering Mechanics (2013).

[56] S. Jin, X. Wen, Hamiltonian-preserving schemes for the Liouville equation with discontinuous potentials, Communications in Mathematical Sciences 3 (2005) 285-315.

[57] S. Jin, X. Wen, Computation of transmissions and reflections in geometrical optics via the reduced Liouville equation, Wave Motion 43 (2006) 667-688.

[58] M. Razi, P. J. Attar, P. Vedula, Adaptive finite difference solutions of Liouville equations in computational uncertainty quantification, Submitted to Reliability Engineering \& System Safety (2013).

[59] C. Shu, S. Osher, Efficient implementation of essentially non-oscillatory shock-capturing schemes, II, Journal of Computational Physics 83 (1989) 32-78.

[60] K. Lika, T. G. Hallam, Traveling wave solutions of a nonlinear reactionadvection equation, Journal of Mathematical Biology 38 (1999) 346-358.

[61] G. Bluman, S. Kumei, On invariance properties of the wave equation, Journal of Mathematical Physics 28 (1987) 307-318. 


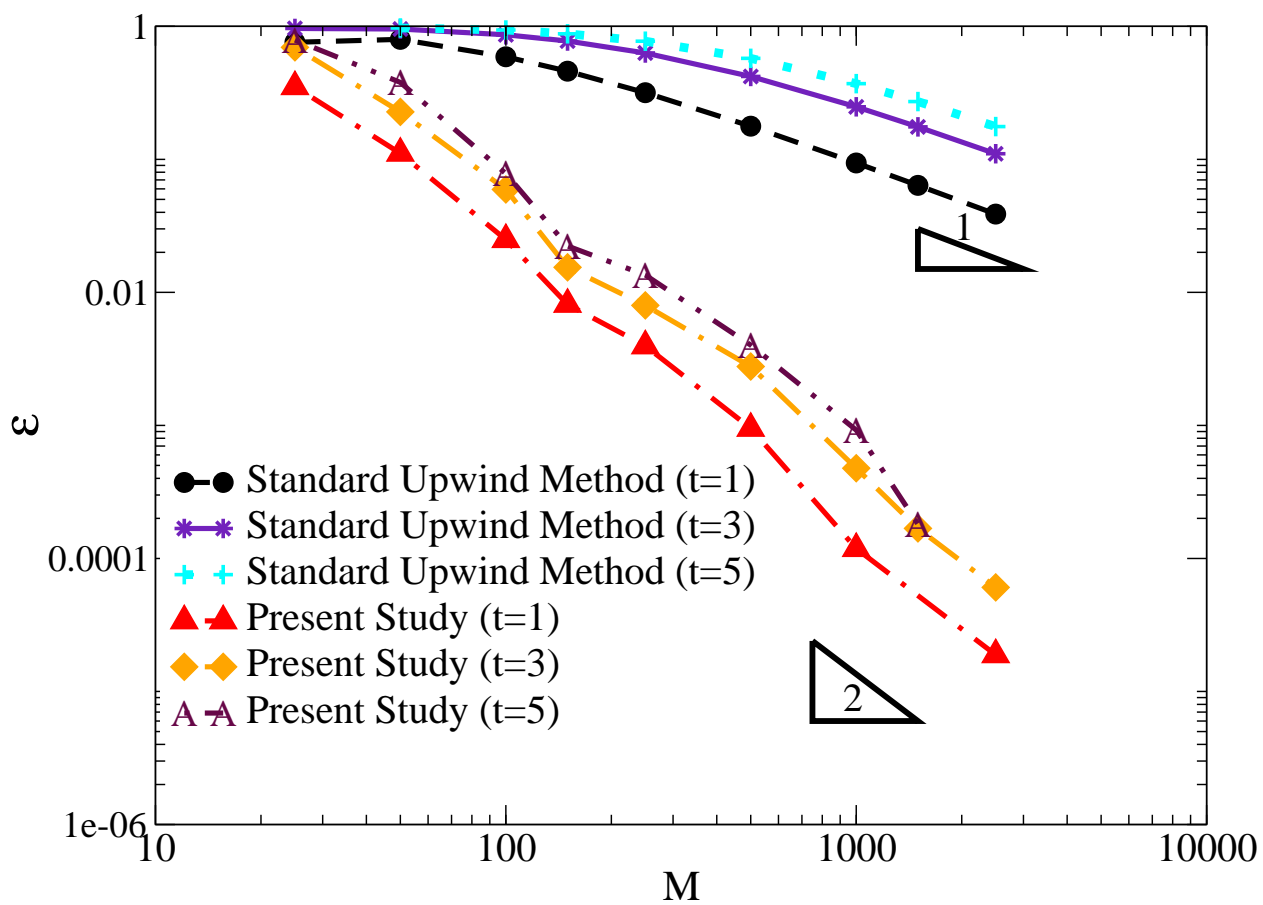

Figure 1: Error in the numerical solution for test problem 1; case with the linear drift function $(D(x)=-x)$ at times $t=1,3$ and 5 versus the number of grid points, $M$. Numerical solutions obtained from the standard first order upwind finite difference method are compared with those obtained using grid adaptation and non-iterative defect correction (Present Study). In the computation of the error, the analytical solution is considered as the reference solution. 


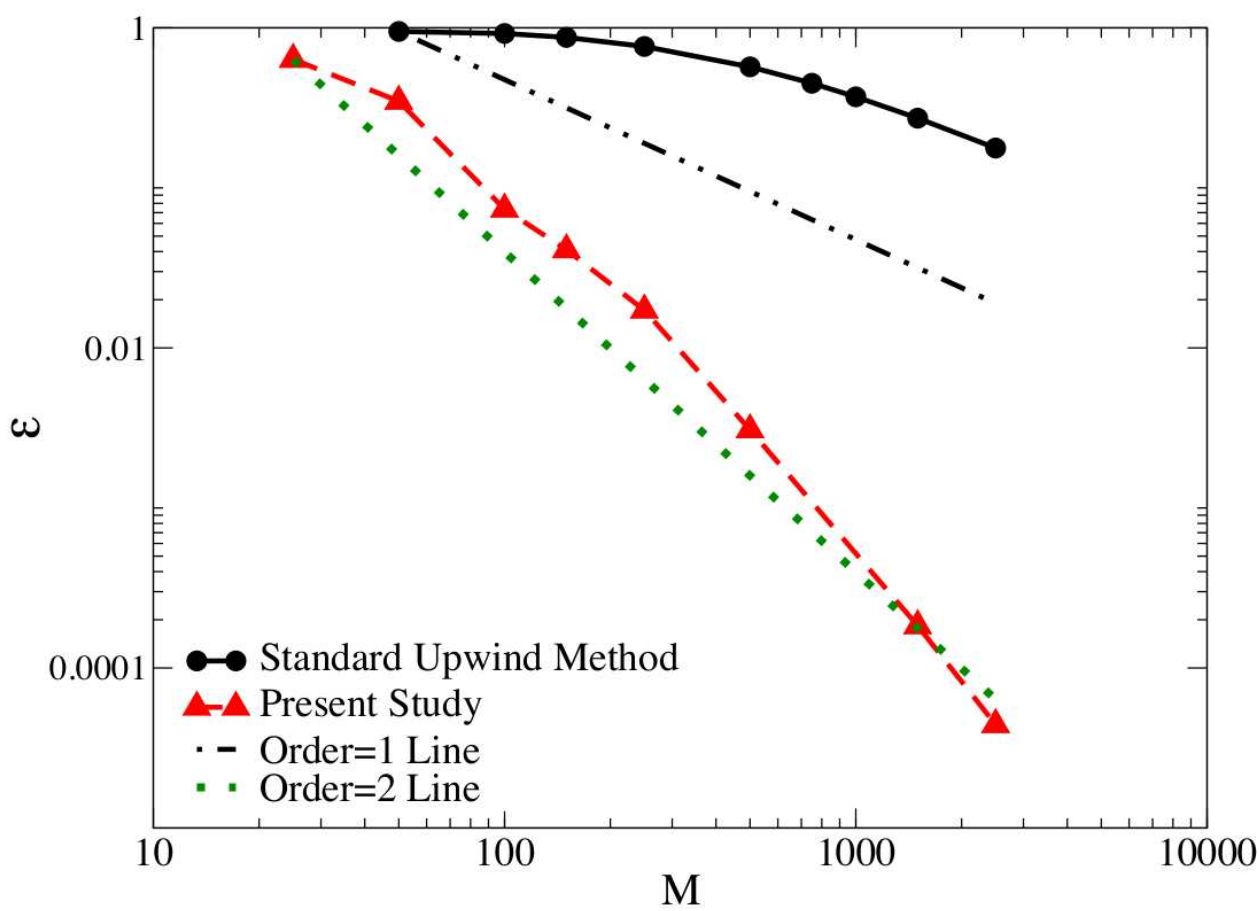

Figure 2: Error in the numerical solution for test problem 1; case with the nonlinear drift function $\left(D(x)=x-x^{3}\right)$ at $t=1$ versus the number of grid points, $M$. Numerical solutions obtained from the standard first order upwind finite difference method are compared with those obtained using grid adaptation and non-iterative defect correction (Present Study). In the computation of the error, the analytical solution is considered as the reference solution. 


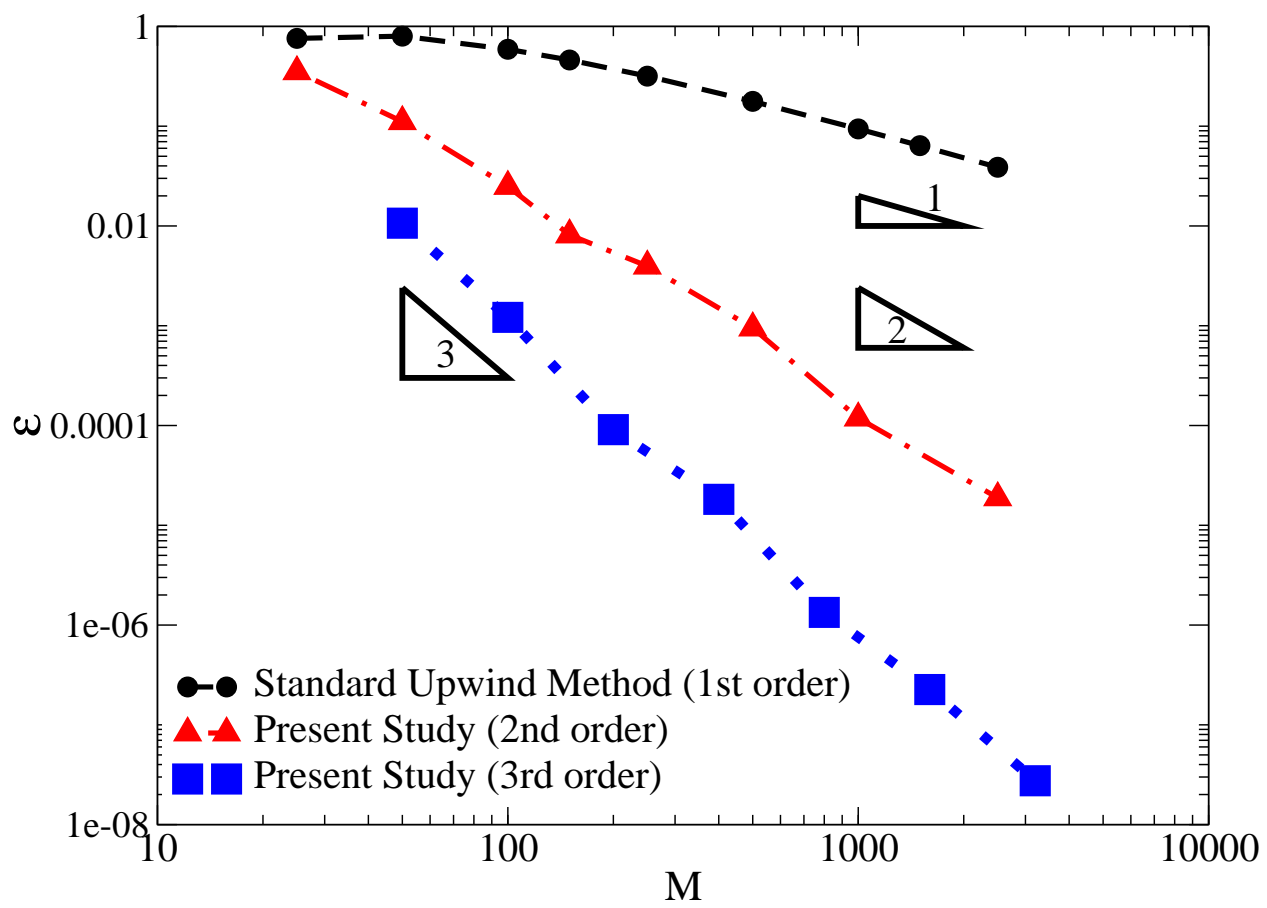

Figure 3: Error in the numerical solution for test problem 1; case with the nonlinear drift function $(D(x)=-x)$ at $t=1$ versus the number of grid points, $M$. The impact of successive modification obtained using grid adaptation and non-iterative defect correction (Present Study) on the accuracy of the solution is shown. In the computation of the error, the analytical solution is considered as the reference solution. 


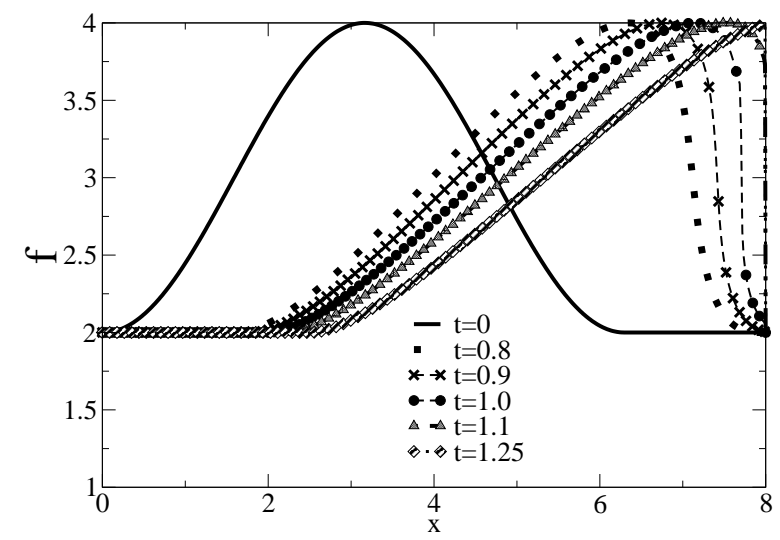

Figure 4: Snapshots, at different points in time, of the spatial distribution of the Burgers equation solution (test problem 2). Results computed using third order ENO with $M=500$.

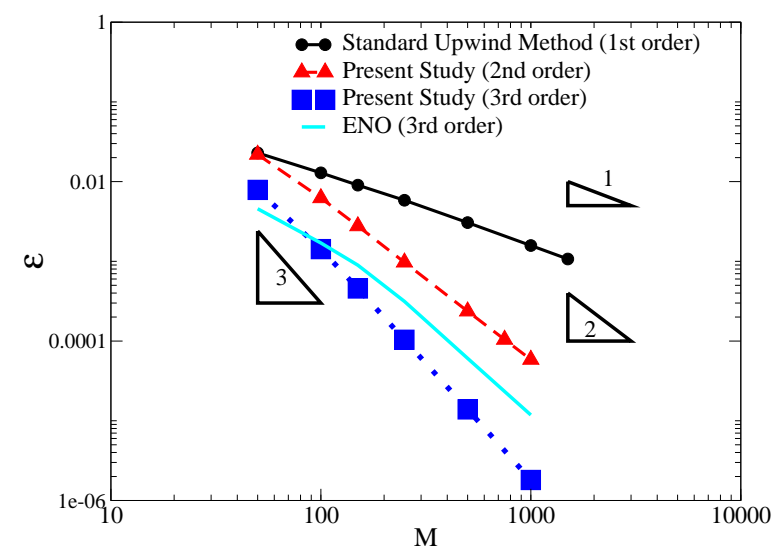

Figure 5: Error in the numerical solution for test problem 2 at $t=0.8$ versus the number of grid points, $M$. Numerical solutions obtained from the standard first order upwind finite difference method are compared with those obtained using grid adaptation and non-iterative defect correction (Present Study). In the computation of the error, the analytical solution is considered as the reference solution. 


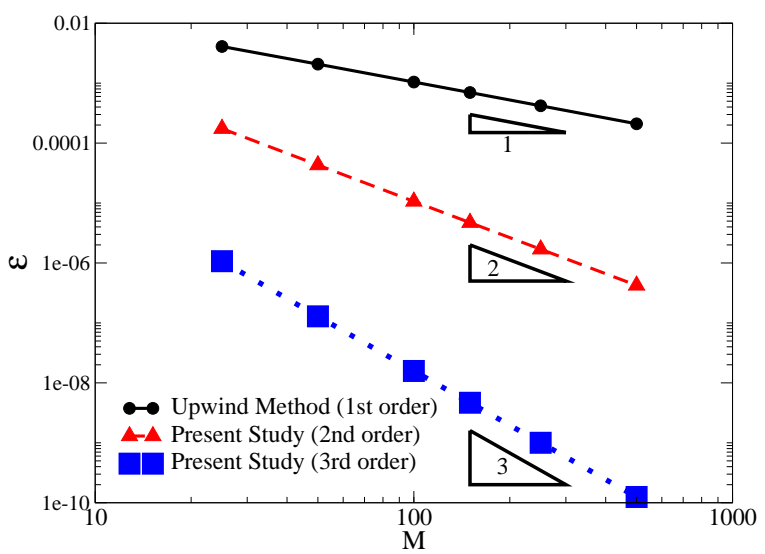

Figure 6: Error in the numerical solution for test problem 3 at $t=1$ versus the number of grid points, $M$. Numerical solutions obtained from the standard first order upwind finite difference method are compared with those obtained using grid adaptation and non-iterative defect correction (Present Study). In the computation of the error, the analytical solution is considered as the reference solution.

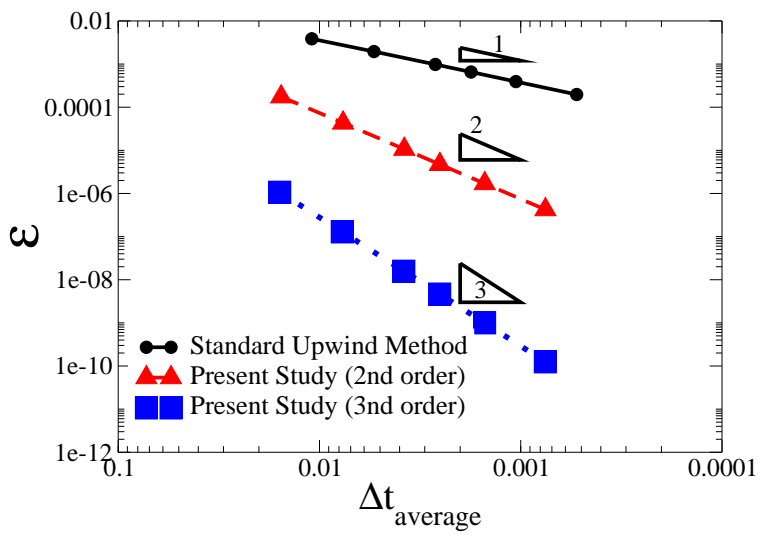

Figure 7: Error in the computed solution for test problem 3 at $t=1$ versus the averaged time-step, $\Delta t_{\text {average. }}$. Numerical solutions obtained from the standard first order upwind finite difference method are compared with those obtained using grid adaptation and non-iterative defect correction (Present Study). In the computation of the error, the analytical solution is considered as the reference solution. 


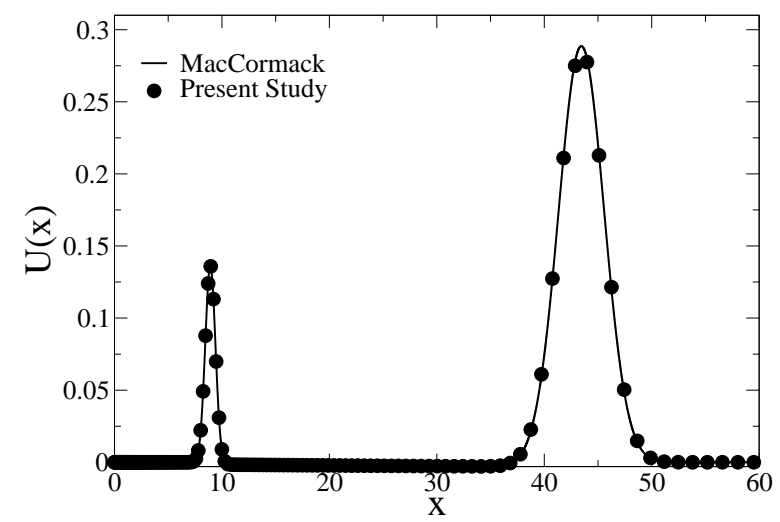

Figure 8: Spatial distribution, at $t=0.75$, of the numerical solution for the second order one-dimensional wave equation with spatially dependent wave speed (Test Problem 4) represented here using a system of two PDEs. Results are compared for the proposed approach with $M=500$ (Present Study) and a highly resolved solution using standard MacCormack with $M=6.4 \times 10^{4}$. (MacCormack)

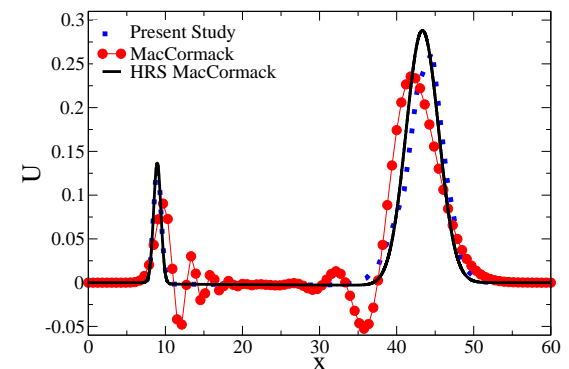

(a) $M=100$

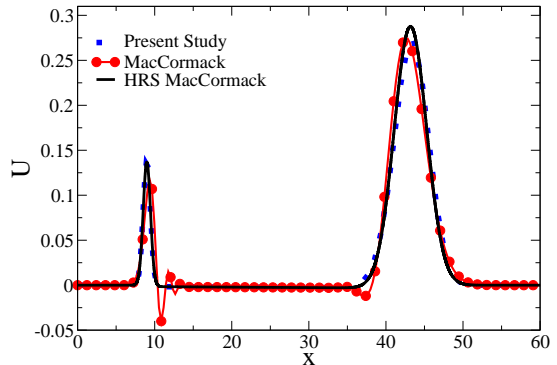

(b) $M=200$

Figure 9: Spatial distribution, at $t=0.75$, of the numerical solution for the second order one-dimensional wave equation with spatially dependent wave speed (Test Problem 4) represented here using a system of two PDEs. Results are given for the proposed approach (Present Study) and MacCormack (MacCormack) with $M=100$ and $M=200$ and compared to a highly resolved MacCormack Solution (HRS MacCormack) with $M=6.4 \times 10^{4}$. 